Edward G. Shaw, Wake Forest School of Medicine, Winston-Salem, NC; Meihua Wang, Radiation Therapy Oncology Group, Philadelphia, PA; Stephen W. Coons, Barrow Neurologic Institute; David G. Brachman, Arizona Oncology Services Foundation, Phoenix, AZ; Jan C. Buckner and Paul D. Brown, Mayo Clinic, Rochester, MN; Keith J. Stelzer, Mid-Columbia Medical Center, Celilo Cancer Center, The Dalles, OR; Geoffrey R. Barger, Wayne State University School of Medicine, Detroit, Ml; Mark R. Gilbert, MD Anderson Cancer Center, Houston, TX; and Minesh P. Mehta, Feinberg School of Medicine, Northwestern University, Chicago, IL.

Submitted April 5, 2011; accepted May 24, 2012; published online ahead of print at www.jco.org on July 30, 2012.

Supported by Radiation Therapy Oncology Group Grant No. U10 CA21661 and Community Clinical Oncology Program Grant No. U10 CA37422 from the National Cancer Institute.

This manuscript's contents are solely the responsibility of the authors and do not necessarily represent the official views of the National Cancer Institute.

Authors' disclosures of potential conflicts of interest and author contributions are found at the end of this article.

Corresponding author: Edward G. Shaw, MD, MA, Department of Radiation Oncology, Wake Forest University School of Medicine, 2000 W. First St, Winston-Salem, NC 27104; e-mail: eshaw@wakehealth.edu.

() 2012 by American Society of Clinical Oncology

0732-183X/12/3025-3065/\$20.00

DOI: $10.1200 / J C O .2011 .35 .8598$

\title{
Randomized Trial of Radiation Therapy Plus Procarbazine, Lomustine, and Vincristine Chemotherapy for Supratentorial Adult Low-Grade Glioma: Initial Results of RTOG 9802
}

Edward G. Shaw, Meihua Wang, Stephen W. Coons, David G. Brachman, Jan C. Buckner, Keith J. Stelzer, Geoffrey R. Barger, Paul D. Brown, Mark R. Gilbert, and Minesh P. Mehta

\section{$\begin{array}{llllllll}\text { A } & \text { B } & \text { S } & \text { T } & \text { R } & \text { A } & \text { C } & \text { T }\end{array}$}

\section{Purpose}

A prior Radiation Therapy Oncology Group (RTOG) clinical trial in anaplastic oligodendroglioma suggested a progression-free survival benefit for procarbazine, lomustine, and vincristine (PCV) chemotherapy in addition to radiation therapy (RT), as have smaller trials in low-grade glioma (LGG).

\section{Patients and Methods}

Eligibility criteria included supratentorial WHO grade 2 LGG, age 18 to 39 years with subtotal resection/biopsy, or age $\geq 40$ years with any extent resection. Patients were randomly assigned to RT alone or RT followed by six cycles of PCV. Survival was compared by using the modified Wilcoxon and log-rank tests.

\section{Results}

In all, 251 patients were accrued from 1998 to 2002. Median overall survival (OS) time and 5-year OS rates for RT versus RT + PCV were 7.5 years versus not reached and $63 \%$ versus $72 \%$, respectively (hazard ratio $[\mathrm{HR}] ; 0.72 ; 95 \% \mathrm{Cl}, 0.47$ to $1.10 ; P=.33$; log-rank $P=.13$ ). Median progression-free survival (PFS) time and 5-year PFS rates for RT versus RT + PCV were 4.4 years versus not reached and $46 \%$ versus $63 \%$, respectively (HR, $0.6 ; 95 \% \mathrm{Cl}, 0.41$ to $0.86 ; P=.06$; $\log$-rank $P=.005)$. OS and PFS were similar for all patients between years 0 and 2 . After 2 years, OS and PFS curves separated significantly, favoring RT + PCV. For 2-year survivors $(n=211)$, the probability of OS for an additional 5 years was $74 \%$ with RT + PCV versus $59 \%$ with RT alone (HR, $0.52 ; 95 \% \mathrm{Cl}, 0.30$ to 0.90 ; log-rank $P=.02$ ).

\section{Conclusion}

PFS but not OS was improved for adult patients with LGG receiving RT + PCV versus RT alone. On post hoc analysis, for 2-year survivors, the addition of PCV to RT conferred a survival advantage, suggesting a delayed benefit for chemotherapy.

\section{J Clin Oncol 30:3065-3070. (C) 2012 by American Society of Clinical Oncology}

\section{INTRODUCTION}

Low-grade gliomas (LGGs) are primary brain tumors classified as grade 1 or 2 by the $\mathrm{WHO}^{1}$; they occur primarily in children and young to intermediate-age adults. The most common LGGs in adults are the WHO grade 2 diffuse astrocytomas, oligodendrogliomas, and mixed oligoastrocytomas. There are approximately 1,800 LGGs diagnosed annually in the United States, representing roughly $10 \%$ of newly diagnosed primary brain tumors. ${ }^{2,3}$ Although WHO grade 1 and 2 tumors are lumped together under the category of LGGs, they are distinctly different neoplasms. Pilocytic astrocytomas, generally well-circumscribed histologi- cally and radiographically, are amenable to cure with gross total resection. In contrast, the diffuse astrocytomas, oligodendrogliomas, and mixed oligo-astrocytomas are infiltrative and thus are less likely to be completely resected and surgically cured. ${ }^{4}$

Over the last 15 years, a number of prospective, randomized US and European clinical trials in adults with supratentorial LGGs have been completed and/or published addressing three key issues in the management of this disease: (1) timing of RT (ie, Should RT be given immediately postoperatively or delayed until time of progression?), (2) dose of RT (ie, Assuming RT is given, is there a local control or survival advantage for higher rather than lower 
doses of RT?), and (3) value of single-agent nitrosourea or temozolomide chemotherapy (ie, Can overall survival [OS] and progressionfree survival [PFS] be improved by adding chemotherapy to RT?). These studies concluded the following: (1) there is no significant difference in OS but there is an improvement in PFS with immediate postoperative versus delayed radiation therapy $(\mathrm{RT})^{5}$; (2) low-dose RT (45 to 50.4 Gy) results in similar OS and PFS compared with high-dose RT (59.4 to 64.8 Gy), ${ }^{6,7}$ although high-dose RT is associated with a higher complication rate ${ }^{7}$; and (3) lomustine chemotherapy plus RT does not improve OS versus RT alone. ${ }^{8}$ The impact of temozolomide remains inadequately defined to date. ${ }^{9,10}$ Nonrandomized trials of procarbazine, lomustine, and vincristine (PCV) chemotherapy as neoadjuvant treatment of LGGs have demonstrated objective responses, prompting the desire to test this regimen in a prospective randomized trial. ${ }^{11}$ Therefore, in 1998, the Radiation Therapy Oncology Group (RTOG), North Central Cancer Treatment Group (NCCTG), Southwest Oncology Group (SWOG), and Eastern Cooperative Oncology Group (ECOG) opened protocol 9802 for adults with supratentorial LGGs. Patients were dichotomized into two risk groups: favorable (age 18 to 39 years with surgeon-defined gross total resection of their tumor) and unfavorable (age $\geq 40$ years, or with subtotal resection or biopsy, irrespective of age). Patients in the favorable-risk group were observed postoperatively; the results of this cohort have been recently published. ${ }^{12}$ Patients in the unfavorablerisk group were randomly assigned to RT alone or RT followed by multiagent chemotherapy using PCV, similar to RTOG Protocol 9402 in which the median PFS time and 5-year PFS rate were significantly improved with the addition of PCV chemotherapy to RT in adults with supratentorial anaplastic oligodendroglioma and oligo-astrocytoma. ${ }^{13}$ This report will focus on the results of an adult supratentorial LGG cohort with unfavorable risk, exploring the putative benefit of PCV chemotherapy added to conventional RT compared with RT alone.

\section{PATIENTS AND METHODS}

\section{Eligibility and Ineligibility Criteria}

Eligibility criteria included histologically proven uni- or multifocal WHO grade 2 astrocytoma, oligodendroglioma, or mixed oligo-astrocytoma based on central pathology review by the neuropathology coprincipal investigator of the study (S.W.C.); any patient age 40 years or older, irrespective of extent of resection, and all patients age 18 to 39 years with a subtotal resection or biopsy; Karnofsky performance score (KPS) $\geq 60$; no, mild, or moderate neurologic symptoms and signs; supratentorial tumor location; operative report as well as pre- and postoperative magnetic resonance imaging (MRI) scan with contrast available for central review; and signed consent form. Patients had to be entered onto the study within 12 weeks of surgery.

Ineligibility criteria were LGG histologies including pilocytic astrocytoma, subependymal giant-cell astrocytoma, pleomorphic xanthroastrocytoma, ganglioglioma, or dysneuroembryoplastic epithelial tumor; presence of WHO grade 3 or 4 glioma on central pathology review; nonsupratentorial tumor location; tumors involving the optic chiasm and/or optic nerve(s); evidence of spread to noncontiguous cranial or spinal leptomeninges; gliomatosis cerebri; synchronous malignancy excluding carcinoma of the cervix in situ or nonmelanomatous skin cancer; prior malignancy unless disease-free for more than 5 years; and prior RT or chemotherapy.

\section{Patient Evaluation and Follow-Up}

Baseline assessment required before registration onto the study included a history and physical (including neurologic) examination, documentation of neurologic symptoms and signs, medications such as steroids and anticonvulsants, KPS, neurologic function score, and a mini-mental status examination. Pre- and postoperative MRI scans, including T1 images without and with contrast, as well as T2 (and when available, the fluid-attenuated inversionrecovery [FLAIR]) images, were required. The maximum preoperative tumor diameter, based on the axial and/or coronal T2 or FLAIR magnetic resonance (MR) images, was measured and recorded at the time of study entry, as was the presence or absence of contrast enhancement, based on the T1 MR images with contrast. The extent of surgical resection was based on the neurosurgeon's assessment as recorded in the operative report. In addition, the study principal investigator (E.G.S.) compared the pre- and postoperative MRI scans to quantify the amount of residual tumor on the postoperative MRI scan. Peripheral blood and paraffin-embedded tumor tissue were collected and stored for future study. Following registration, patients were observed, with serial clinical evaluations and MRI scans every 6 months, which is conventional for this disease. Tumor progression was defined as a clear increase in the T2 or FLAIR abnormality and/or new contrast enhancement.

\section{$R T$ and Chemotherapy}

All patients received the same RT whether they were randomly assigned to receive RT alone or RT plus chemotherapy. The radiation dose was $54 \mathrm{~Gy}$ given in 30 fractions of 1.8 Gy each (prescribed to isocenter) over 6 weeks. The treatment fields included the T2 or FLAIR MRI-defined tumor volume plus a $2-\mathrm{cm}$ margin to block edge, resulting in an approximate $1-\mathrm{cm}$ dosimetric margin. Patients randomly assigned to receive chemotherapy were treated with six cycles of postradiation procarbazine $\left(60 \mathrm{mg} / \mathrm{m}^{2}\right.$ orally per day on days 8 through 21 of each cycle), lomustine $\left(110 \mathrm{mg} / \mathrm{m}^{2}\right.$ orally on day 1 of each cycle), and vincristine $\left(1.4 \mathrm{mg} / \mathrm{m}^{2}\right.$ [maximum $2 \mathrm{~g}$ ]) intravenously on days 8 and 29 of each cycle. The cycle length was 8 weeks. Salvage treatment at the time of tumor progression was permitted on an individualized basis.

\section{Statistical Considerations}

The primary end point was OS, and secondary end points included PFS and grade $\geq 3$ toxicity according to version 2.0 of the National Cancer Institute Common Toxicity Criteria. ${ }^{14}$ Eligible patients were stratified by age ( $<40 v \geq 40$ years), KPS (60 to $80 v 90$ to 100), presence of enhancement on the preoperative MRI scan (present $v$ absent), and histology (astrocytoma or astrocytoma-dominant mixed-glioma $v$ oligodendroglioma or oligodendroglioma-dominant mixed glioma), then randomly assigned to either RT alone or RT + PCV in a permuted block design by using the method described by Zelen. ${ }^{15}$

The study was designed to accrue 120 eligible patients per arm giving $90 \%$ power to detect a relative $21 \% 5$ - year OS increase (to $85 \%$ ) for the RT + PCV arm compared with the RT alone arm, assuming 5-year OS rate of 70\% with RT alone. This survival difference corresponds to a hazard ratio (HR) of 0.46 for RT + PCV versus RT alone. The study was designed to test the survival difference by using a modified Wilcoxon test ${ }^{16}$ at the overall significance level of 0.05 . Three blinded interim analyses were planned (and occurred) by the RTOG Data Monitoring Committee to occur after 20, 40, and 60 deaths, with specified stopping rules; in each instance, the $P$ value was greater than the prespecified early reporting criterion, suggesting that the null hypothesis could not be rejected, and the study continued. The significance level for the final analysis will be 0.0478 when each patient has been observed for a minimum of 5 years or after 80 deaths have occurred.

Frequency distributions of the patient pretreatment characteristics for the two treatment arms were compared by using $\chi^{2}$ tests. The $Z$ test was used to test for differences in binomial proportions of severe or worse treatment toxicities between the two arms. For the OS and PFS end points, the KaplanMeier method ${ }^{17}$ was used to estimate the rates, and the Wilcoxon test ${ }^{16}$ was used to compare differences between the two treatment arms. Survival differences were also assessed by using the log-rank test, ${ }^{18}$ which is more sensitive to late separation. An event for OS was death due to any cause. An event for PFS was the first reported occurrence of tumor progression or death. The Cox proportional hazards mode ${ }^{19}$ was used to estimate the HR associated with each end point. The assumption of proportional hazards was assessed by testing the interaction of treatment and time $(P<.05)$. Because the nonproportional hazards and hazards cross at approximately 2 years, the ad hoc analyses for patients surviving to 2 years were also performed. Prognostic 


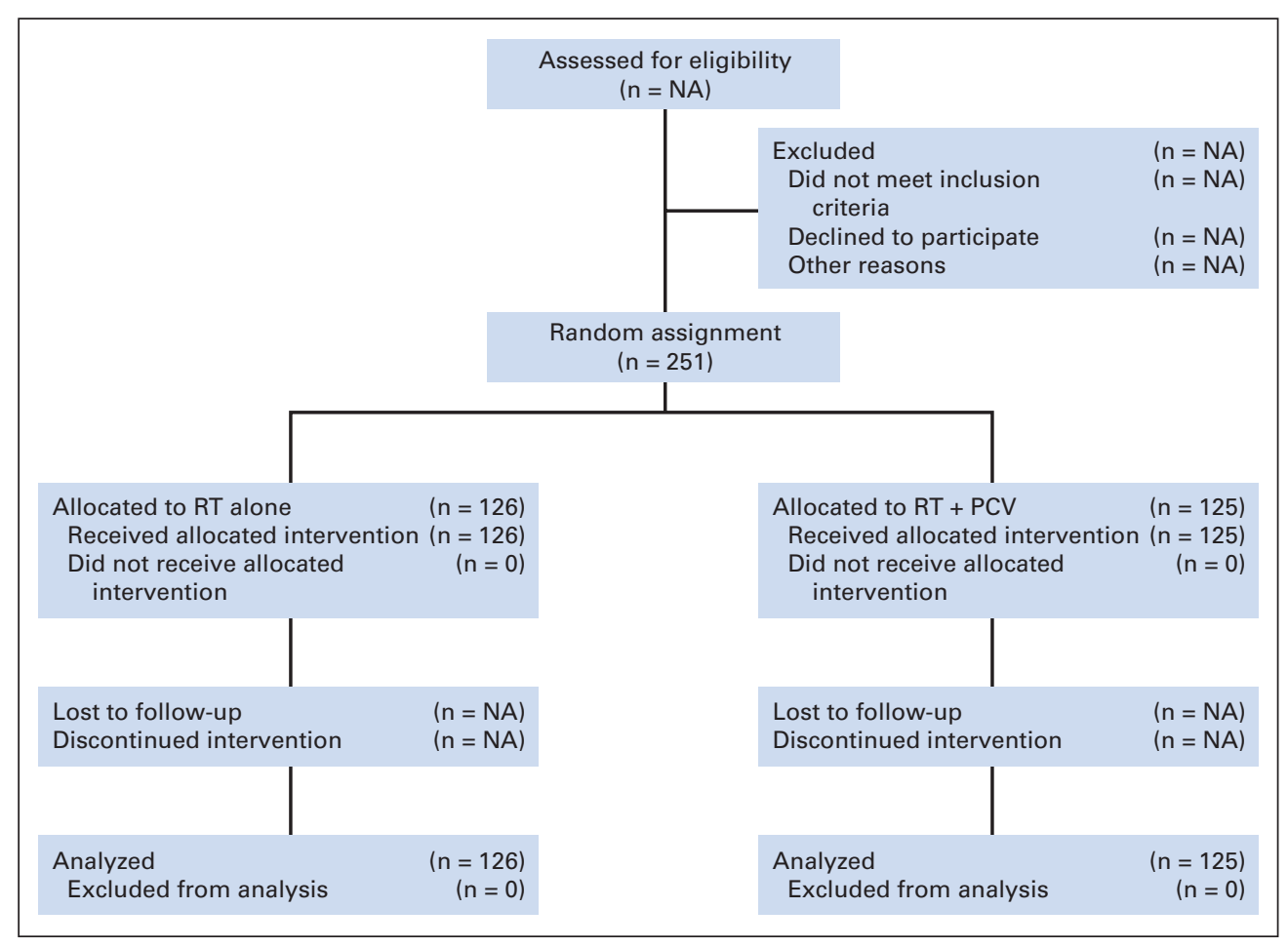

Fig 1. CONSORT diagram. NA, not assessed; PCV, procarbazine, lomustine, and vincristine; RT, radiation therapy.

factors were analyzed for their effect on OS and PFS by using uni- and multivariate analysis and included age, sex, KPS, neurologic symptoms and signs, contrast enhancement, extent of surgical resection, and histology.

\section{RESULTS}

\section{Accrual, Follow-Up, and Patient Characteristics}

Between 1998 and 2002, 251 patients were registered onto the study and were randomly assigned: 126 patients to the RT alone arm and 125 to the RT + PCV arm (Fig 1). At the time of this report, 88 (35\%) of the 251 patients had died. Median follow-up time for all patients still alive is 5.9 years. Patient characteristics for patients on each treatment arm are provided in Table 1. Treatment compliance (ie, treatment with RT or RT + PCV per protocol or with an acceptable variation) was $95 \%$ to $97 \%$.

\section{Survival for All Registered and Randomly Assigned Patients}

The median OS time was not reached (more than 8.5 years) for the RT + PCV arm; it was 7.5 years (95\% CI, 6.0 to not reached) for RT alone, whereas the 2- and 5-year OS rates were $85 \%$ and $72 \%$ for patients who received RT + PCV versus $87 \%$ and $63 \%$ for those who received RT alone (HR, $0.72 ; 95 \% \mathrm{CI}, 0.47$ to $1.10 ; P=$ .33 ; log-rank $P=.13)$. The median PFS time was not reached (more than 6.1 years) for the RT + PCV arm; it was 4.4 years (95\% CI, 3.6 to 5.9 years) for RT alone, whereas the 2- and 5-year PFS rates were $74 \%$ and $63 \%$ for patients who received RT + PCV versus $75 \%$ and $46 \%$ for those who received RT alone (HR, 0.6; 95\% CI, 0.41 to $0.86 ; P=.06$; $\log$-rank $P=.005)$. In the initial 2 -year follow-up phase, the OS curves for the two regimens actually cross, as illustrated in Figure 2; a similar trend is observed for PFS as illustrated in Figure 3.

\section{Post Hoc Analysis of Survival for Patients Surviving to 2 Years}

Of the initially registered and randomly assigned 251 patients, $211(84 \%)$ survived to 2 years. OS and PFS rates were similar for patients treated with RT + PCV or RT alone between years 0 and 2, although crossing of the curves does occur. However, beyond 2 years, the survival curves separated significantly, with both OS and PFS favoring patients treated with PCV + RT. When the $16 \%$ of patients who died (34) or were censored (six) within 2 years are removed from the analysis, the conditional probability of OS for the remaining 211 patients who survived for 2 years to survive for an additional 3 and 5 years was $84 \%$ and $74 \%$ with RT + PCV compared with $72 \%$ and $59 \%$ with RT alone (HR, 0.52; 95\% CI,

\begin{tabular}{|c|c|c|c|c|}
\hline \multirow[b]{2}{*}{ Characteristic } & \multicolumn{2}{|c|}{ RT Arm } & \multicolumn{2}{|c|}{$\begin{array}{c}\mathrm{RT}+\mathrm{PCV} \\
\text { Arm }\end{array}$} \\
\hline & No. & $\%$ & No. & $\%$ \\
\hline \multicolumn{5}{|l|}{ Age, years } \\
\hline Median & \multicolumn{2}{|c|}{40} & \multicolumn{2}{|c|}{41} \\
\hline Range & \multicolumn{2}{|c|}{$22-79$} & \multicolumn{2}{|c|}{$18-82$} \\
\hline Median tumor size, $\mathrm{cm}$ & \multicolumn{2}{|c|}{5.0} & \multicolumn{2}{|c|}{4.7} \\
\hline KPS 90-100 & & 74 & & 75 \\
\hline Gross total resection & & 9 & & 11 \\
\hline \multicolumn{5}{|l|}{ Histology } \\
\hline Astrocytoma & & 23 & & 29 \\
\hline Oligodendroglioma & & 45 & & 40 \\
\hline Mixed astrocytoma/oligodendroglioma & & 32 & & 31 \\
\hline Enhancement: yes & & 60 & & 65 \\
\hline \multicolumn{5}{|c|}{$\begin{array}{l}\text { Abbreviations: KPS, Karnofsky performance score; PCV, procarbazine, lomus- } \\
\text { tine, and vincristine; RT, radiation therapy. }\end{array}$} \\
\hline
\end{tabular}




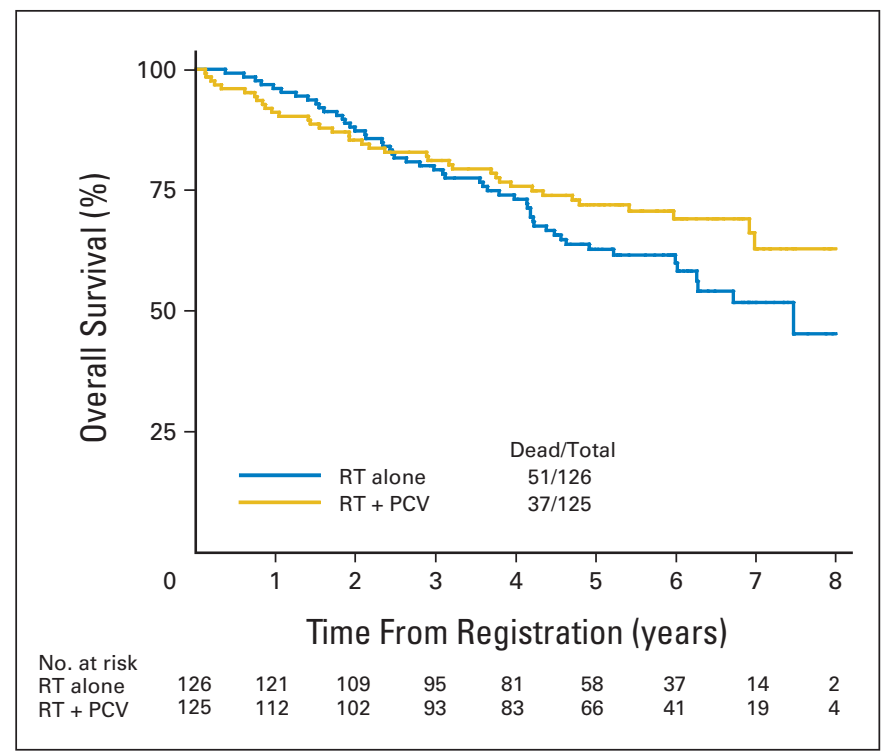

Fig 2. Overall survival for all patients from date of registration/random assignment. PCV, procarbazine, lomustine, and vincristine; RT, radiation therapy.

0.30 to 0.90 ; $\log$-rank $P=.02)$; comparable data for PFS were $74 \%$ and $66 \%$ with RT + PCV versus $52 \%$ and $37 \%$ with RT alone (HR, 0.44 ; $95 \%$ CI, 0.28 to 0.69 ; $\log$-rank $P<.001)$. The HR for RT + PCV versus RT alone, adjusted for histology, was 0.52 for death (95\% CI, 0.30 to $0.91 ; P=.02$ ) and 0.45 for progression $(95 \% \mathrm{CI}$, 0.29 to $0.70 ; P<.001)$. The characteristics of the 2 -year survivors were compared with characteristics of those who died within 2 years: the 2-year survivors were significantly more likely to be younger than age 40 years $(51 \% v 20 \% ; P<.001)$, have undergone gross total or subtotal resection instead of biopsy only $(56 \% v 33 \%$; $P=$ .006), and have either oligodendroglioma or oligodendrogliomadominant mixed glioma histology (61\% v35\%; $P=.003$; Figs 4 and 5).

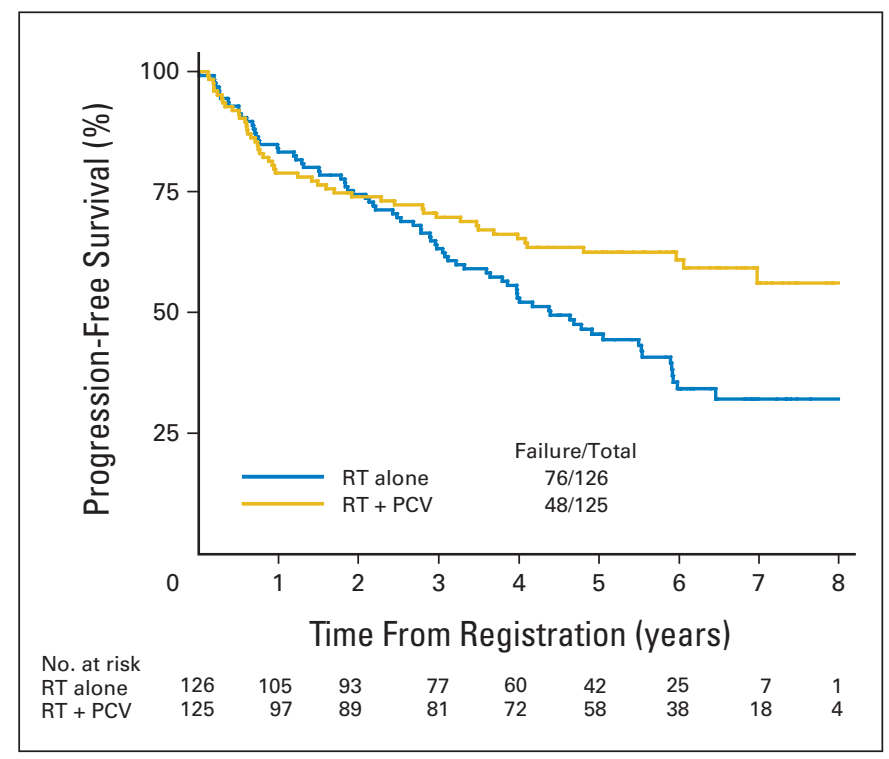

Fig 3. Progression-free survival for all patients from date of registration/ random assignment. PCV, procarbazine, lomustine, and vincristine; RT, radiation therapy.

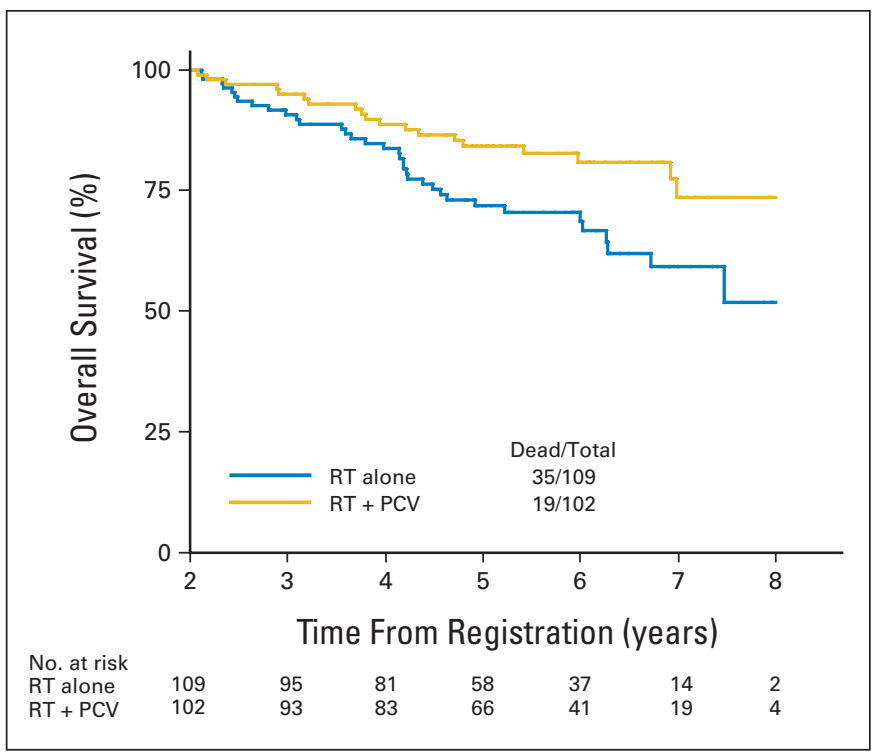

Fig 4. Overall survival for patients surviving to 2 years. PCV, procarbazine, lomustine, and vincristine; RT, radiation therapy.

\section{Toxicity of Treatment}

The incidence of grade 3 and 4 hematologic toxicity was $8 \%$ and $3 \%$ with RT alone compared with $51 \%$ and $15 \%$ with RT + PCV $(P<.001)$. There were no chemotherapy- or RT-related grade 5 toxicities. The incidence of late grade $3 \mathrm{RT}$ toxicity was $1 \%$ to $2 \%$. No late grades 4 to $5 \mathrm{RT}$ or chemotherapy toxicities were reported.

\section{Treatment of Tumor Recurrence and Failure Patterns}

In all, 103 patients (41\%) had tumor recurrence; when evaluated by treatment arm, 65 of 103 recurrences were seen in the RT alone arm ( $63 \%$ of all recurrences; $52 \%$ of all patients on the RT arm) and 38 of 103 recurrences were seen in the RT + PCV arm (37\% of all recurrences;

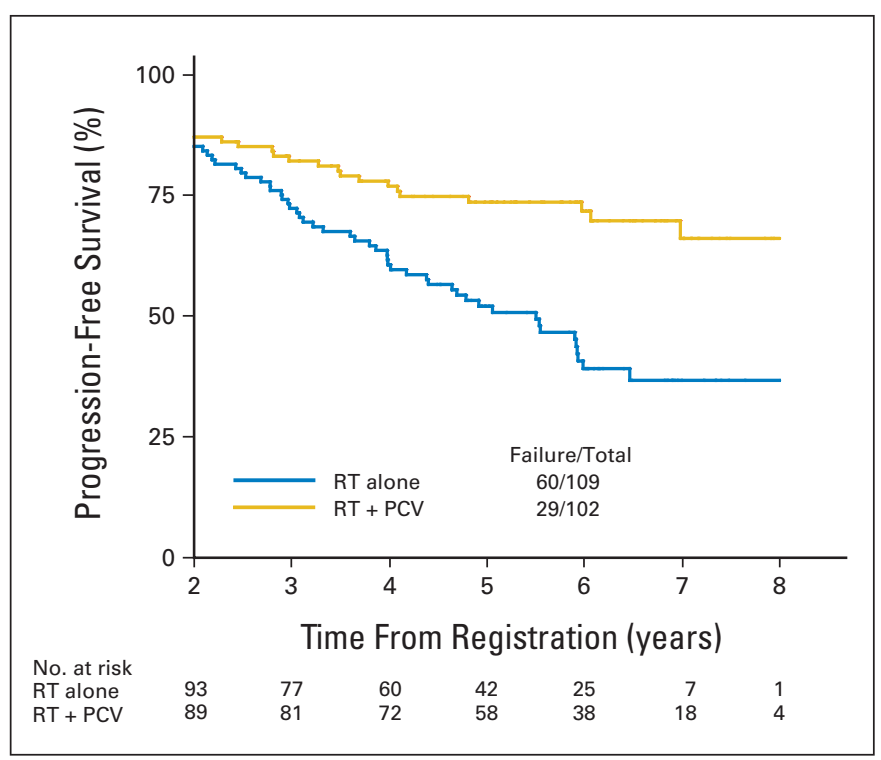

Fig 5. Progression-free survival for patients surviving to 2 years. PCV, procarbazine, lomustine, and vincristine; RT, radiation therapy. 
$30 \%$ of all patients on the RT + PCV arm). Salvage treatment included surgery in $25 \%$ of RT alone and $32 \%$ of RT + PCV patients, chemotherapy in $71 \%$ of RT alone and $53 \%$ of RT + PCV patients (primarily temozolomide), and additional RT (fractionated or stereotactic radiosurgery) in $23 \%$ of RT alone and $13 \%$ of RT + PCV patients.

\section{DISCUSSION}

From the perspective of the primary end point, RT + PCV did not improve OS versus RT alone; therefore, the trial is negative. A positive trial would have required a shift in 5-year survival from 70\% to 85\% (a relative increase of $21 \%$ ); in reality, the 5 -year survival values were $63 \%$ versus $72 \%$, a relative increase of $14 \%$. In terms of secondary end points, this study demonstrated improvement in PFS for patients treated with RT + PCV versus RT alone, similar to the results of RTOG 9402 trial in adult supratentorial anaplastic oligodendroglioma and oligo-astrocytoma. ${ }^{13}$

In the post hoc subset analysis, the addition of PCV to RT conferred an advantage to both OS and PFS, reducing the risk of death by $48 \%$ and progression by $56 \%$, with the recognition that the curves for both end points do cross. These data suggest a possible delayed survival benefit for chemotherapy. There are some putative explanations for this observation: (1) PCV is only of benefit for true LGGs (ie, excluding LGG with undiagnosed anaplastic features that behave more aggressively), and (2) despite random assignment, the molecular features of the two cohorts by treatment arm might in fact be different and unbalanced, so that a subpopulation of patients relatively more sensitive to PCV chemotherapy ended up being enrolled on the combination arm (we are proceeding with molecular profiling to better understand this, including analysis of $\mathrm{O}^{6}$-methylguanine-DNA methyltransferase [MGMT], 1p19q, and PTEN promoter methylation, among others). Furthermore, since patients surviving 2 years and beyond tended to have more complete resections and oligodendroglial histology, perhaps this is the subset that derives therapeutic benefit with RT + PCV.

Because RTOG Protocols 9402 and 9802 were developed in the 1990s, PCV was used rather than temozolomide, which has since become part of the standard of care for glioblastoma. ${ }^{20}$ Though there are no randomized trials in LGG comparing temozolomide to other agents, a number of phase II nonrandomized open-label studies have been published in which temozolomide was used either at the time of recurrence (progression following surgery, RT, with or without non-temozolomide chemotherapy $)^{21-23}$ or in newly diagnosed disease ${ }^{9,10}$ (Table 2). With the exception of one study, ${ }^{21}$ typical doses of $200 \mathrm{mg} / \mathrm{m}^{2}$ per day for 5 days every 28 days for six to 12 cycles resulted in no complete responses, and partial response rates varied from $10 \%$ to $47 \%$. In recurrent disease, the 12 -month PFS rate was $39 \%$ in one series ${ }^{22}$ and $73 \%$ to $75 \%$ in two others, ${ }^{21,23}$ suggesting at least modest activity for temozolomide in recurrent LGG, but the large variability of outcomes in the recurrent setting brings into question the issue of malignant transformation as a key variable influencing PFS, perhaps even more so than therapeutic effect. In patients with newly diagnosed LGG, the 3-year PFS and OS rates were $57 \%$ to $66 \%$ and $81 \%$ to $81 \%$, respectively. ${ }^{9,10}$ These results are similar to the 3 -year PFS and OS rates observed in this study with RT alone (3-year PFS, 63\%; 3-year OS, 79\%) and RT + PCV (3-year PFS, 70\%; 3-year OS,
Table 2. Temozolomide Chemotherapy for Low-Grade Glioma

\begin{tabular}{|c|c|c|c|c|c|c|c|}
\hline \multirow[b]{3}{*}{ Reference } & \multirow[b]{3}{*}{$\begin{array}{l}\text { No. of } \\
\text { Patients }\end{array}$} & \multirow[b]{3}{*}{ Setting } & \multirow{2}{*}{\multicolumn{2}{|c|}{$\begin{array}{r}\text { Disease } \\
\text { Response } \\
(\%)\end{array}$}} & \multicolumn{3}{|c|}{ Survival (\%) } \\
\hline & & & & & \multirow{2}{*}{$\begin{array}{l}12- \\
\text { Month } \\
\text { PFS }\end{array}$} & \multirow[b]{2}{*}{$\begin{array}{l}\text { 3-Year } \\
\text { OS }\end{array}$} & \multirow[b]{2}{*}{$\begin{array}{l}\text { 3-Year } \\
\text { PFS }\end{array}$} \\
\hline & & & CR & PR & & & \\
\hline Quinn, et al $\left.\right|^{21}$ & 46 & $\mathrm{R}$ & 24 & 37 & 76 & & \\
\hline Pace, et al ${ }^{22}$ & 43 & $\mathrm{R}$ & 0 & 47 & 39 & & \\
\hline Tosoni, et al ${ }^{23}$ & 30 & $\mathrm{R}$ & 0 & 30 & 73 & & \\
\hline Brada, et al ${ }^{9}$ & 30 & $\mathrm{~N}$ & 0 & 10 & & 82 & 66 \\
\hline Kesari, et al ${ }^{10}$ & 44 & $\mathrm{~N}$ & 0 & 20 & & 81 & 57 \\
\hline \multicolumn{8}{|c|}{$\begin{array}{l}\text { Abbreviations: CR, complete response; N, newly diagnosed; OS, overal } \\
\text { survival; PFS, progression-free survival; PR, partial response; } R \text {, recurrent. }\end{array}$} \\
\hline
\end{tabular}

$81 \%$ ), suggesting that initial temozolomide versus RT is a reasonable clinical question to test in newly diagnosed LGG. In fact, the European Organisation for Research and Treatment of Cancer (EORTC) has recently completed a randomized trial comparing postoperative temozolomide alone $\left(75 \mathrm{mg} / \mathrm{m}^{2}\right.$ per day for 21 days, every 28 days until progression) to RT alone $(50.4 \mathrm{~Gy})$ in adults with newly diagnosed high-risk supratentorial LGG. Patients are stratified by whether they have chromosome 1p loss of heterozygosity (LOH). A further important question would be the therapeutic value of RT + temozolomide compared with RT alone. The RTOG piloted and completed the RT + temozolomide arm in the RTOG 0424 phase II trial; now, an intergroup trial performed by ECOG, RTOG, NCCTG, and SWOG is comparing RT alone to RT plus concurrent and post-RT temozolomide. The study opened in fall 2009 and as of October 2011, has accrued 53 of 540 patients. Together, these trials will potentially provide answers regarding the value of temozolomide alone versus RT alone and RT plus temozolomide versus RT in this patient population.

Jenkins et $\mathrm{al}^{24}$ have published data regarding the impact of $1 p 19 q$ LOH on outcome in 98 patients with newly diagnosed LGG. Those with $1 p 19 q \mathrm{LOH}$ had a median survival time of 11.9 years, significantly better than the 8.1-year median survival time for those without $1 p 19 q \mathrm{LOH}^{24}$ Analyses of progression and survival as a function of $1 p 19 q \mathrm{LOH}$ status and other molecular signatures, including, for example, PTEN promoter methylation and $I D H$ expression, are ongoing in patients treated on RTOG Protocol 9802. Further, the results of this study will be reanalyzed over the upcoming years, because the possibility exists that with time, the HR might reach statistical significance for an OS benefit, permitting rejection of the null hypothesis.

In conclusion, for this study, PFS but not OS was improved for adults with newly diagnosed supratentorial LGG receiving RT + PCV versus RT alone. Beyond 2 years, the addition of PCV to RT conferred an advantage for both OS and PFS in a post hoc analysis of a subset and reduced the risk of death by $48 \%$ and progression by $56 \%$, suggesting a possible delayed benefit for chemotherapy.

\section{AUTHORS' DISCLOSURES OF POTENTIAL CONFLICTS OF INTEREST}

Although all authors completed the disclosure declaration, the following author(s) and/or an author's immediate family member(s) indicated a 
financial or other interest that is relevant to the subject matter under consideration in this article. Certain relationships marked with a " $U$ " are those for which no compensation was received; those relationships marked with a " $C$ " were compensated. For a detailed description of the disclosure categories, or for more information about ASCO's conflict of interest policy, please refer to the Author Disclosure Declaration and the Disclosures of Potential Conflicts of Interest section in Information for Contributors.

Employment or Leadership Position: Minesh P. Mehta, Pharmacyclics (C) Consultant or Advisory Role: Mark R. Gilbert, Genentech (C), Merck (C); Minesh P. Mehta, Adnexus (C), Bayer (C), Genentech (C), Merck (C), TomoTherapy (C) Stock Ownership: Minesh P. Mehta, Pharmacyclics, TomoTherapy Honoraria: Mark R. Gilbert, Merck, Genentech, Abbott Laboratories Research Funding: None Expert Testimony: None Other Remuneration: None

\section{AUTHOR CONTRIBUTIONS}

Conception and design: Edward G. Shaw, Geoffrey R. Barger, Jan C. Buckner, Stephen W. Coons, Mark R. Gilbert, Minesh P. Mehta Provision of study materials or patients: Edward G. Shaw, Geoffrey R. Barger, David G. Brachman, Paul D. Brown, Jan C. Buckner, Mark R. Gilbert, Minesh P. Mehta, and Keith J. Stelzer

Collection and assembly of data: Edward G. Shaw, Meihua Wang Data analysis and interpretation: Edward G. Shaw, Minesh P. Metha, Meihua Wang

Manuscript writing: All authors

Final approval of manuscript: All authors

\section{REFERENCES}

1. Kleihues $P$, Burger PC, Scheithauer BW: Histological Typing of Tumours of the Central Nervous System (ed 2). Berlin, Germany, Springer, 1993

2. Central Brain Tumor Registry of the United States (CBTRUS): Statistical Report: Primary Brain Tumors in the United States, 1998-2002. Hinsdale, IL, CBRTUS, 2006. http://www.cbtrus.org/reports/20052006/2006report.pdf

3. Jemal A, Siegel R, Ward E, et al: Cancer statistics, 2006. CA Cancer J Clin 56:106-130, 2006

4. Brown PD, Shaw EG: Low-grade gliomas, in Gunderson LL, Tepper JE (eds): Clinical Radiation Oncology (ed 2). Philadelphia, PA, Churchill-Livingstone, 2006, pp 493-514

5. van den Bent MJ, Afra D, de Witte O, et al: Long-term efficacy of early versus delayed radiotherapy for low-grade astrocytoma and oligodendroglioma in adults: The EORTC 22845 randomised trial. Lancet 366:985-990, 2005

6. Karim AB, Maat B, Hatlevoll R, et al: A randomized trial on dose-response in radiation therapy of low-grade cerebral glioma: European Organization for Research and Treatment of Cancer Study (EORTC) Study 22844. Int J Radiat Oncol Biol Phys 36:549-556, 1996

7. Shaw $E$, Arusell $R$, Scheithauer $B$, et al: $A$ prospective randomized trial of low- versus highdose radiation therapy in adults with supratentorial low-grade glioma: Initial report of a North Central Cancer Treatment Group/Radiation Therapy Oncology Group/Eastern Cooperative Oncology Group Study. J Clin Oncol 20:2267-2276, 2002
8. Eyre HJ, Crowley JJ, Townsend JJ, et al: A randomized trial of radiotherapy versus radiotherapy plus CCNU for incompletely resected low-grade gliomas. A Southwest Oncology Group study. J Neurosurg 78:909-914, 1993

9. Brada M, Viviers L, Abson C, et al: Phase ॥ study of primary temozolomide chemotherapy in patients with WHO grade II gliomas. Ann Oncol 14:1715-1721, 2003

10. Kesari S, Schiff D, Drappatz J, et al: Phase ॥ study of protracted daily temozolomide for low-grade gliomas in adults. Clin Cancer Res 15:330-337, 2009

11. Buckner JC, Gesme D Jr, O'Fallon JR, et al: Phase II trial of procarbazine, lomustine, and vincristine as initial therapy for patients with low-grade oligodendroglioma or oligoastrocytoma: Efficacy and associations with chromosomal abnormalities. J Clin Oncol 21:251-255, 2003

12. Shaw EG, Berkey B, Coons SW, et al: Recurrence following neurosurgeon-determined grosstotal resection of adult supratentorial low-grade glioma: Results of a prospective clinical trial. J Neurosurg 109:835-841, 2008

13. Intergroup Radiation Therapy Oncology Group Trial 9402, Cairncross G, Berkey B, et al: Phase III trial of chemotherapy plus radiotherapy compared with radiotherapy alone for pure and mixed anaplastic oligodendroglioma: Intergroup Radiation Therapy Oncology Group Trial 9402. J Clin Oncol 24:2707-2714, 2006

14. Cancer Therapy Evaluation Program: Common Toxicity Criteria, Version 2.0. Revised March 23, 1998. http://ctep.cancer.gov/protocolDevelopment/electronic_ applications/docs/ctcv20_4-30-992.pdf
15. Zelen M: The randomization and stratification of patients to clinical trials. J Chron Dis 27:365-375, 1974

16. Klein J, Moeschberger M: Survival Analysis: Techniques for Censored and Truncated Data. New York, NY, Springer, 2003

17. Kaplan EL, Meier P: Nonparametric estimation from incomplete observations. J Am Stat Assoc 53:457-481, 1958

18. Mantel N: Evaluation of survival data and two new rank order statistics arising in its consideration. Cancer Chemother Rep 50:163-170, 1966

19. Cox DR: Regression models and life-tables. J R Stat Soc B 34:187-220, 1972

20. Stupp R, Mason WP, van den Bent MJ, et al: Radiotherapy plus concomitant and adjuvant temozolomide for glioblastoma. N Engl J Med 352:987996, 2005

21. Quinn JA, Reardon $D A$, Friedman $A H$, et al: Phase II trial of temozolomide in patients with progressive low-grade glioma. J Clin Oncol 21:646651, 2003

22. Pace A, Vidiri A, Galiè E, et al: Temozolomide chemotherapy for progressive low-grade glioma: Clinical benefits and radiological response. Ann Oncol 14:1722-1726, 2003

23. Tosoni $A$, Franceschi $E$, Ermani $M$, et al: Temozolomide three weeks on and one week off as first line therapy for patients with recurrent or progressive low grade gliomas. J Neurooncol 89:179185, 2008

24. Jenkins $R B$, Blair $H$, Ballman $K V$, et al: $A$ $t(1 ; 19)(q 10 ; p 10)$ mediates the combined deletions of $1 p$ and $19 q$ and predicts a better prognosis of patients with oligodendroglioma. Cancer Res 66: 9852-9861, 2006 